\title{
$15 \%$ Azelainsäure in Hydrogel zur Therapie der papulopustulösen Rosazea
}

\section{Kowalzick \\ D. Mischke}

\author{
15\% Azelaic Acid Hydrogel in the Topical Treatment of Papulopustular Rosacea
}

\section{Zusammenfassung}

Kürzlich wurde über das gute therapeutische Ansprechen von Patienten mit papulopustulöser Rosazea auf eine topische Therapie mit $15 \%$ Azelainsäure in Hydrogel berichtet. Wir berichten über 4 Patienten mit papulopustulöser Rosazea, die 2-mal täglich für bis zu 12 Wochen mit Skinoren ${ }^{\circledR} 15 \%$ Gel behandelt wurden. Bei allen 4 behandelten Patienten kam es binnen 4 Wochen zu einer Besserung des Befundes. Am Ende der Behandlung war der klinische IGA-Score durchschnittlich auf $61 \%$ des Ausgangswertes gesunken. Besonders gut sprachen die entzündlichen Hautveränderungen (Papeln und Pusteln) auf die Therapie an (Besserung des Scores auf 44\%), gefolgt von der Besserung des Erythems (63\%). Die Wirkung auf die Teleangiektasien war nur gering (74\%). Alle Patienten tolerierten die Behandlung gut, lokale Nebenwirkungen wurden nicht beobachtet.

\section{Abstract}

Recently, the good therapeutic effect of $15 \%$ acelaic acid hydrogel in patients with papulopustular rosacea was reported. We report on 4 patients with papulopustular rosacea, treated twice daily for up to 12 weeks with Skinoren ${ }^{\circledR} 15 \%$ gel. In all 4 treated patients the skin improved within 4 weeks. At the end of the observation period the mean clinical IGA score had dropped to $61 \%$ of baseline. Especially the inflammatory lesions (papules and pustules) responded well to treatment (44\%), followed by the improvement of erythema (63\%). The therapeutic effect on teleangiectasia was only weak ( $74 \%$ of baseline). All patients tolerated the treatment well and no local side effects occurred.

\section{Einleitung}

Die Rosazea ist eine chronische entzündliche Hauterkrankung des Gesichtes, gekennzeichnet durch Flush, persistierende Erytheme, Teleangiektasien, Papeln und Pusteln sowie Talgdrüsenund Bindegewebshyperplasie (Phymbildung). Das Stadium 2 der Erkrankung ist gekennzeichnet durch persistierende Erytheme, Teleangiektasien sowie Papeln und Pusteln [8]. Die Ätiologie der Erkrankung ist unklar, vermutlich spielt eine Regulationsstörung der Blutgefäße des Gesichtes eine wesentliche Rolle [4]. Topische und systemische Antibiotika wie Metronidazol und Minocyclin sind in der Behandlung der Rosacea papulopustulosa erfolgreich [9]. Beim Wirkmechanismus sind wahrscheinlich weniger die antimikrobiellen Effekte der Antibiotika als ihre antiinflammatorischen auf neutrophile Granulozyten von Bedeutung [2]. Azelainsäure hat neben einer antimikrobiellen Wirkung eine antientzündliche durch Hemmung der Generation von reaktiven Sauerstoffspezies durch neutrophile Granulozyten [1] und immunmodulierende Effekte auf T-Lymphozyten. Die Substanz wirkt daneben auch antikomedogen. In einer 20\%igen Creme (Skinoren ${ }^{\circledR}$ Creme) ist die Substanz seit über 10 Jahren zur Behandlung der Acne vulgaris zugelassen. Der positive Effekt dieser 
Tab. 1 Klinischer IGA (Investigator's Global Assessment)-Score zur Bewertung des Schweregrades bei papulopustulöser Rosazea (Rosazea Stadium 2) nach [10].

\begin{tabular}{|c|c|c|c|c|c|c|c|}
\hline Grad & $\begin{array}{l}0 \\
\text { frei }\end{array}$ & $\begin{array}{l}1 \\
\text { minimal }\end{array}$ & $\begin{array}{l}2 \\
\text { mild }\end{array}$ & $\begin{array}{l}3 \\
\text { mild bis } \\
\text { moderat }\end{array}$ & $\begin{array}{l}4 \\
\text { moderat }\end{array}$ & $\begin{array}{l}5 \\
\text { moderat bis } \\
\text { schwer }\end{array}$ & $\begin{array}{l}6 \\
\text { schwer }\end{array}$ \\
\hline Papeln und Pusteln & keine & kaum & wenige & deutlich & betont & viele (groß) & $\begin{array}{l}\text { sehr viele } \\
\text { (konfl.) }\end{array}$ \\
\hline Erythem & $\begin{array}{l}\text { kein bis } \\
\text { Rest }\end{array}$ & $\begin{array}{l}\text { Rest bis } \\
\text { mild }\end{array}$ & mild & $\begin{array}{l}\text { mild bis } \\
\text { moderat }\end{array}$ & moderat & $\begin{array}{l}\text { moderat bis } \\
\text { schwer }\end{array}$ & $\begin{array}{l}\text { moderat bis } \\
\text { schwer }\end{array}$ \\
\hline Teleangiektasien & $\begin{array}{l}\text { keine bis } \\
\text { mild }\end{array}$ & $\begin{array}{l}\text { mild bis } \\
\text { moderat }\end{array}$ & $\begin{array}{l}\text { mild bis } \\
\text { moderat }\end{array}$ & $\begin{array}{l}\text { mild bis } \\
\text { moderat }\end{array}$ & $\begin{array}{l}\text { mild bis } \\
\text { moderat }\end{array}$ & moderat & $\begin{array}{l}\text { moderat bis } \\
\text { schwer }\end{array}$ \\
\hline
\end{tabular}

Spezialität auch auf die papulopustulöse Rosazea konnte in einer Doppelblindstudie [3] nachgewiesen werden. In der Folge wurde ein lipidarmes (<3\%) Hydrogel mit 15\% Azelainsäure (Skinoren ${ }^{\circledR}$ $15 \%$ Gel) entwickelt, das auch zur Therapie der Acne papulopustulosa des Gesichts zugelassen ist. Trotz niedrigerer Konzentration erreicht das Hydrogel verglichen mit der Creme eine deutlich höhere Konzentration des Wirkstoffes in der Haut [5]. Kürzlich wurden zwei Studien publiziert, die die gute Wirksamkeit von Azelainsäure in 15 \%igem Hydrogel bei der papulopustulösen Rosazea zeigte [10]. Wir berichten über unsere Erfahrungen mit Skinoren ${ }^{\circledR} 15 \%$ Gel bei 4 Patienten mit papulopustulöser Rosazea.

\section{Patienten und Methoden}

\section{Patienten und Therapie}

Behandelt wurden je 2 weibliche und männliche Patienten (Altersdurchschnitt: 59 Jahre) mit langjährig bestehender, zuletzt untherapierter papulopustulöser Rosazea (Stadium 2 der Rosazea). Ein Patient hatte zusätzlich ein Rhinophym (Stadium 3 der Rosazea). Bei diesem Patienten wurde vor Beginn der Lokaltherapie eine OP des Rhinophyms mittels hochtouriger Schleifung durchgeführt. Alle Patienten wurden angewiesen, 2-mal täglich Skinoren ${ }^{\circledR}$ 15\% Gel (Schering Deutschland GmbH, Berlin) auf ihre Gesichtshaut dünn aufzutragen. Die Behandlungsdauer war bis zu 12 Wochen vorgesehen.

\section{Evaluation des Behandlungserfolges}

Zur Bewertung der Erkrankungsaktivität der Rosacea papulopustulosa verwendeten wir den klinischen IGA (Investor's Global Assessment)-Score (Tab.1) zur Bewertung von entzündlichen Läsionen, Erythem und Teleangiektasien [10]. Der IGA-Score wurde vor Therapiebeginn und dann alle 4 Wochen bis zu Ende der Beobachtungszeit ermittelt. Darüber hinaus wurden Fotografien vor Therapiebeginn und am Ende der Beobachtungszeit angefertigt.

\section{Ergebnisse}

Bei allen 4 behandelten Patienten kam es binnen 4 Wochen zu einer Besserung des Befundes. Am Ende der Behandlung war der klinische IGA-Score durchschnittlich auf $61 \%$ des Ausgangswertes gesunken (Abb.1). Besonders gut sprachen die entzündli-

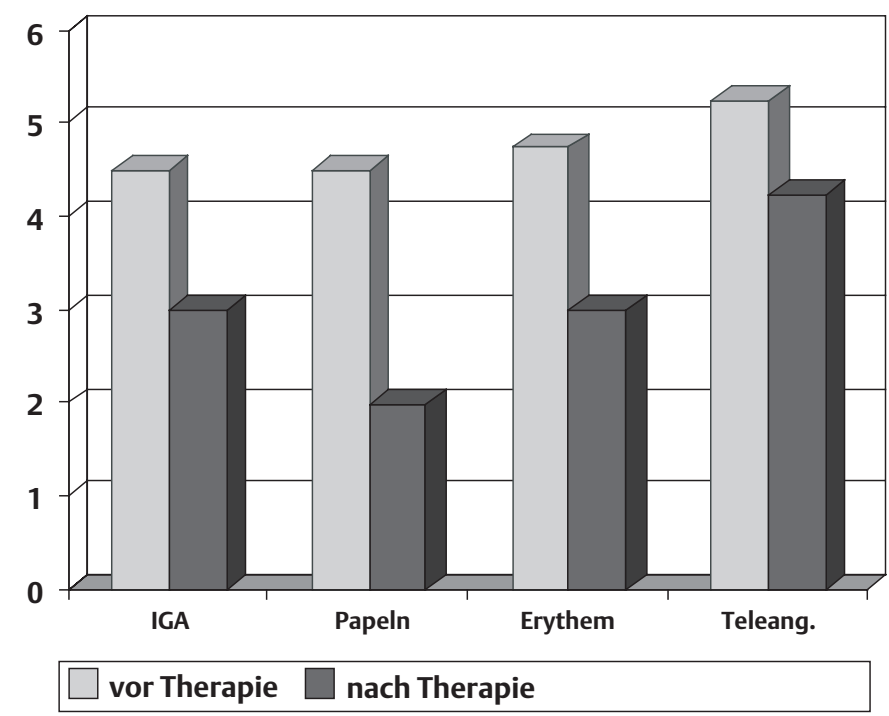

Abb. 1 Durchschnittlicher klinischer IGA-Score, und Teilscores für Papeln und Pusteln, Erythem sowie Teleangiektasien bei vier Patienten mit papulopustulöser Rosazea vor und nach Lokaltherapie mit Azelainsäure 15\% Hydrogel (Skinoren ${ }^{\circledR} 15 \%$ Gel).

chen Hautveränderungen (Papeln und Pusteln) auf die Therapie an (Besserung des Scores auf 44\%) (Abb. 2), gefolgt von der Besserung des Erythems (63\%) (Abb. 2 u.3). Die Wirkung auf die Teleangiektasien war nur gering (74\%) (Abb. 3). Alle Patienten tolerierten die Behandlung gut, lokale Nebenwirkungen, insbesondere Hauttrockenheit und -irritation, wurden nicht beobachtet.

\section{Diskussion}

Eine 20\%ige Azelainsäure-Creme zeigte in einer vehikelkontrollierten randomisierten Doppelblindstudie einen signifikanten Effekt auf entzündliche Läsionen (Reduktion auf 27\% des Ausgangswertes) und auf das Erythem (52\%) bei Rosazea papulopustulosa. Keinerlei Effekt wurde auf die Teleangiektasien beobachtet. Allerdings traten bei $49 \%$ der Patienten lokale Nebenwirkungen auf, speziell brennende Missempfindungen [3]. Gegenüber einer 0,75\%igen Metronidazol-Creme zeigte sich die 20\%ige Azelainsäure-Creme in einer doppelblinden Halbseitenstudie als vergleichbar wirksam bezogen auf Papeln, Pusteln und Erythem [7]. 


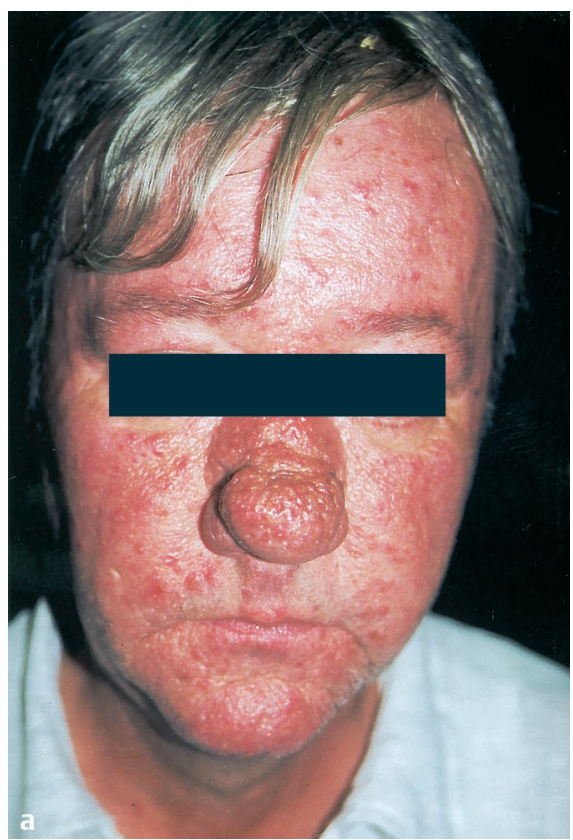

Abb. 2 46-jähriger Patient mit Rosazea und Rhinophym

(Stadium 3) vor (a) und nach (b) 8-wöchiger Lokaltherapie mit Azelainsäure 15\% Hydrogel (Skinoren ${ }^{\circledR}$ 15\% Gel). Deutlicher Rückgang von Papeln und Pusteln und Erythem (entsprechend Rückgang des IGAScores von 5 auf 3). Außerdem wurde vor Therapiebeginn eine operative hochtourige Schleifung des Rhinophyms durchgeführt.
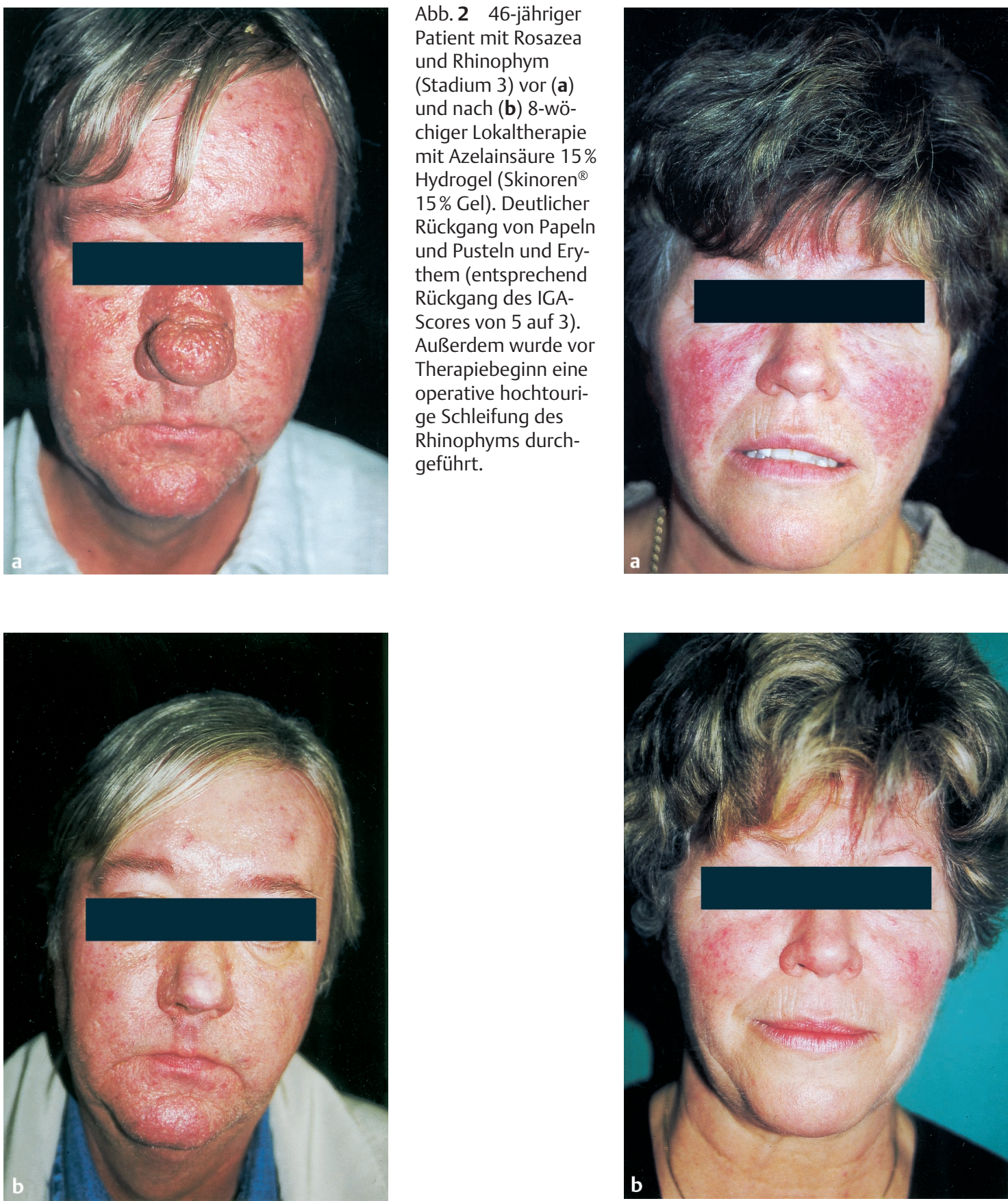

Abb. 3 58-jährige Patientin mit papulopustulöser Rosazea (Stadium 2) vor (a) und nach (b) 12-wöchiger Lokaltherapie mit Azelainsäure 15\% Hydrogel (Skinoren ${ }^{\circledR}$ 15\% Gel). Deutlicher Rückgang von Papeln und Pusteln und teilweiser Rückgang des Erythems. Allenfalls geringer Effekt auf Teleangiektasien. Rückgang des IGAScores von 5 auf 3.

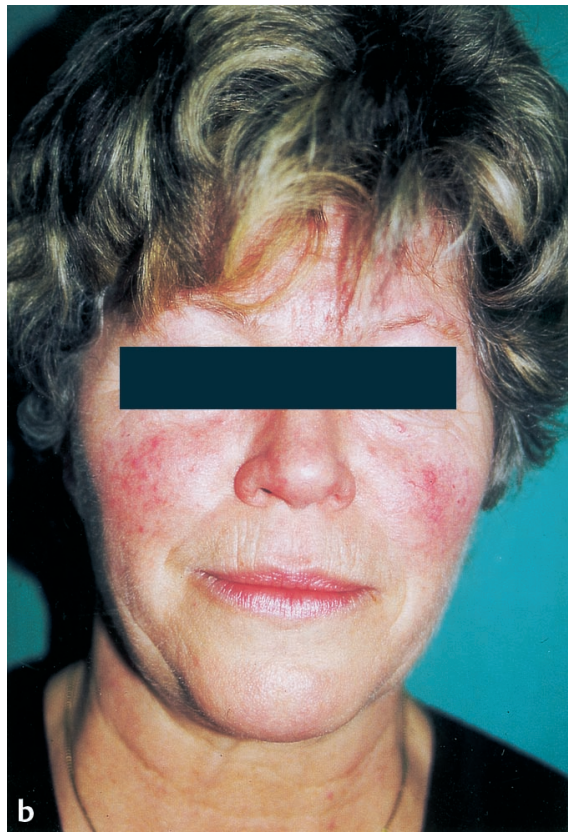

In zwei kürzlich erschienenen Publikationen wird über zwei vehikelkontrollierte randomisierte Doppelblindstudien und eine randomisierte, doppelblinde Vergleichsstudie gegenüber 0,75\%igem Metronidazol-Gel berichtet. 15\%iges AzelainsäureHydrogel zeigte eine signifikante Reduktion von Papeln und Pusteln auf $42 \%$ bzw. $49 \%$ und des Erythems auf 56\% bzw. 54\% des Ausgangsbefundes der Rosacea papulopustulosa. Brennende Missempfindungen traten bei etwa einem Drittel der behandelten Patienten auf, waren aber meist nur leicht und vorübergehend [10]. Gegenüber dem 0,75\%igen Metronidazol-Gel zeigte sich das $15 \%$ ige Azelainsäure-Gel in den ersten 8 Therapiewochen als gleichwertig, nach 12 und 15 Wochen als signifikant überlegen (der Behandlungseffekt stagnierte unter Metronidazol-Gel). Die Zahl der Papeln und Pusteln nahm auf 28\% ab, das Erythem ging auf $41 \%$ des Ausgangswertes zurück. Ein therapeu-

tischer Effekt auf die Teleangiektasien wurde in beiden Behandlungsarmen nicht beobachtet [6].

Trotz der kleinen Fallzahl spiegeln auch unsere Resultate mit Skinoren ${ }^{\circledR} 15 \%$ Gel diese an größeren Patientengruppen mit topischer Azelainsäure bei papulopustulöser Rosazea gemachten Erfahrungen gut wider: Am besten sprechen die entzündlichen Läsionen der Papeln und Pusteln an. Der therapeutische Effekt auf das Erythem ist ebenfalls deutlich vorhanden, jedoch weniger stark ausgeprägt. Auch bei unseren Patienten fand sich eine Besserung dieser beiden Parameter bereits nach 4 Wochen Therapiedauer. Ein eindeutiger Behandlungseffekt auf die Teleangiektasien der Rosazea ist durch diese Lokaltherapie dagegen nicht zu erwarten. Die Verträglichkeit von Azelainsäure 15\%ig in Hydrogel war bei unseren Patienten durchweg gut. Die topische 
Anwendung von Skinoren ${ }^{\circledR} 15 \%$ Gel stellt eine rasch wirksame Behandlungsform der papulopustulösen Rosazea dar.

\section{Literatur}

${ }^{1}$ Akamatsu H, Komura J, Asada Y, Miyachi Y, Niwa Y. Inhibitory effect of azelaic acid on neutrophil functions: a possible cause for its efficacy in treating patogenetically unrelated diseases. Arch Dermatol Res 1991; 283: $162-166$

${ }^{2}$ Akamatsu H, Oguchi M, Nishijima S, Asada Y, Takasashi M, Ushijima T, Niwa Y. The inhibition of free radical generation by human neutrophils through the synergistic effects of metronodazole with palitoleic acid: a possible mechanism of action of metronidazole in rosacea and acne. Arch Dermatol Res 1990; 282: 449-454

${ }^{3}$ Bjerke R, Fyrand O, Graupe K. Double-blind comparison of azelaic acid $20 \%$ cream and its vehicle in treatment of papulo-pustular rosacea. Acta Dermatovenereol (Stockh.) 1999; 79: 456-459

${ }^{4}$ Dahl MV. Pathogenesis of Rosacea. Adv Dermatol 2001; 17: 29-45

${ }^{5}$ Draelos ZD, Graupe K. A new topical formulation of mild to moderate papulopustular rosacea: azelaic acid 15\% gel. American Academy of Dermatology 61th Annual Meeting, San Francisco, California, USA March 21 - 26, 2003 (Poster \# 49)

${ }^{6}$ Elewski BE, Fleischer AB, Pariser DM. A comparison of $15 \%$ azaleic acid gel and $0.75 \%$ metrinidazole gel in the topical treatment of papulopustular rosacea: results of a randomized trial. Arch Dermatol 2003; 139: $1444-1450$

${ }^{7}$ Maddin S. A comparison of topical azelaic acid $20 \%$ cream and topical metronidazole $0.75 \%$ cream in the treatment of patients with papulopustular rosacea. J Am Acad Dermatol 1999; 40: 961 - 965

8 Plewig G, Kligman AM. Acne and Rosacea. 3rd Edition. Berlin: Springer-Verlag, 2000: 460-501

9 Rebora A. The management of rosacea. Am J Clin Dermatol 2002; 3 : $548-587$

10 Thiboutot D, Thieroff-Ekerdt R, Graupe K. Efficacy and safety of acelaic acid (15\%) gel as a new treatment for papulopustular rosacea: results from two vehicle-controlled, randomised phase III studies. J Am Acad Dermatol 2003; 48: 836-845
In Anwesenheit des Vizepräsidenten der Freien Universität Berlin, Herrn Prof. Dr. R. Tauber, wurde am 21. 2. 2004 an der Klinik und Hochschulambulanz für Dermatologie am Campus Benjamin Franklin der Charité - Universitätsmedizin Berlin der von der Berliner Stiftung für Dermatologie vergebene Forschungspreis in Höhe von Euro 15000,00 - dieses Jahr zu gleichen Teilen an Herrn Prof. Dr. Matthias Schmuth, Universitätsklinik für Dermatologie und Venerologie in Innsbruck, und Herrn Prof. Dr. Michael Schön, Rudolf Virchow Zentrum der Universität Würzburg, verliehen. Die Stiftung würdigt mit diesen Preisen die Arbeiten von Herrn Professor Schmuth zur Barrierefunktion der Haut und klinischer Implikationen neuer nukleärer Hormonrezeptoren sowie die Arbeiten von Herrn Professor Schön über die kutane Leukozyten-Rekrutierung als einen Schlüssel zu Pathogenese und Therapie entzündlicher Erkrankungen. Darüber hinaus hat die Berliner Stiftung für Dermatologie, wie auch in den vergangenen Jahren, Ausbildungs- und Weiterbildungsstipendien vergeben. Sie wurden an Frau Dr. Aylin Kalayciyan aus der Dermatologischen Abteilung der Universität Cerrahpa, Istanbul, Türkei, Frau Dr. Ana-Maria Forsea von der Universität Carol Davila in Bukarest, Rumänien, und Herrn Dr. Mebratu Ketema aus Addis Ababa, Äthiopien, überreicht.

Die Forschungsförderung durch Stiftungen ist eine immer wichtiger werdende Aufgabe in Zeiten eingeschränkter staatlicher Forschungsförderungsmittel. Das diesjährige wissenschaftliche Symposium der Berliner Stiftung für Dermatologie, in dessen Rahmen die Übergabe der Preise und Stipendien stattfand, stand unter dem Motto „Die Dermatologie im Spannungsfeld der Molekularen Medizin“.

Die Berliner Stiftung für Dermatologie hat ihren Förderungsschwerpunkt auf dem Gebiet der Dermatologie und ihrer Grenzgebiete mit dem Ziel, dermatologische Forschung unter besonderer Berücksichtigung der Molekularbiologie und Dermatopharmakologie voranzutreiben. Hierdurch soll die Umsetzung neuer Erkenntnisse in die klinische Dermatologie zur verbesserten Erkennung und Versorgung von Hautkrankheiten führen. Die Unterstützung von Forschungsvorhaben und Weiterbildungsstipendien sowie die Förderung des internationalen Austausches von Wissenschaftlerinnen und Wissenschaftlern auf dem Gebiet der dermatologischen Forschung dient der Verwirklichung der Stiftungsziele und spiegelt sich im Spektrum der diesjährig Geförderten wider.

Ein besonderes Ziel der Berliner Stiftung für Dermatologie ist die Förderung von Kolleginnen und Kollegen aus osteuropäischen Ländern. So wird dieses Jahr zusätzlich die Universitäts-Hautklinik in Cluj-Napoca, Rumänien, in Form einer umfangreichen Buchspende unterstützt.

Die Berliner Stiftung für Dermatologie hat ihren Sitz an der Klinik und Hochschulambulanz für Dermatologie am Campus Benjamin Franklin der Charité - Universitätsmedizin Berlin. Weitere Informationen sind über Herrn Prof. Dr. Dr. C. Geilen, Geschäftsführer der Stiftung (Tel. 030/8445-6912), bzw. über die Homepage (www.stiftung-dermatologie.de) erhältlich. 